\title{
Evaluation of the presence of bioaerosols in a neonatal intensive care unit
}

\author{
Wendy Morgado-Gamero \\ Departamento de Ciencias \\ Naturales y Exactas \\ Universidad de la Costa CUC \\ Barranquilla-Atlántico \\ wmorgado1@cuc.edu.co
}

\author{
Martha Mendoza Hernández \\ Departamento de Ingeniería Civil \\ $y$ Ambiental \\ Universidad del Norte \\ Barranquilla-Atlántico \\ mmendoza9309@gmail.com
}

\author{
Dayana Agudelo-Castañeda \\ Departamento de Ingeniería Civil \\ y Ambiental \\ Universidad del Norte \\ Barranquilla -Atlántico \\ mdagudelo@uninorte.edu.co
}

\author{
Margarita Castillo Ramírez \\ Red de Monitoreo de Calidad del \\ Aire de Barranquilla. EPA \\ Barranquilla Verde \\ Barranquilla - Atlántico \\ mcastilloramirez87@gmail.com
}

\author{
Alexander Parody \\ Facultad de Ingenieria \\ Universidad Libre \\ Barranquilla - Atlántico \\ alexander_parody@hotmail.com
}

\author{
Leidi Posso Mendoza \\ Departamento de Bateriologia \\ Universida Metropolitana \\ Barranquilla - Atlántico \\ heidy_posso@unimetro.edu.co
}

\begin{abstract}
In this study, the emmission of the Bacterial bioaerosols in the different respiratory system in a neonatal intensive care unit (ICU). In this study, a six-stage Andersen impactor was used for the sampling of bioaerosols with a flow rate of $28.3 \mathrm{~L} / \mathrm{min}$. The concentrations obtained from bacterial bioaerosols ranged between 67 and $423 \mathrm{CFU} / \mathrm{m3}$, with an average value of 110.13 CFU / m3, which can represent a possible threat to the health of the workers and neonates in the ICU. The results indicated than Staphylococcus saprophyticus and Staphylococcus epidermidis predominated, especially in the fifth and sixth stages, which means second bronchi and alveoli. While Staphylococcus was the most prevalent genus, Alloiococcus otitidis, Bacillus subtiles, Bacillus thuringiensis, Kocuria rosea and Pseudomonas pseudoalcaligene occurred in the alveoli.
\end{abstract}

Keywords- air quality; bioaerosols; Neonatal intensive care unit; respiratory infections; Antibiotic resistance; public health; Risks evaluation.

\section{INTRODUCCIÓN}

La contaminación del aire constituye una de las principales problemáticas ambientales que más impacto o efectos nocivos tiene sobre las personas y su calidad de vida; en las últimas décadas ha quedado demostrada la actuación del aire en la transmisión de enfermedades infecciosas y la dispersión de sustancias nocivas para la salud [1], este medio ha sido considerado como el vehículo más importante en la dispersión de todo tipo de microorganismos, pudiendo estos ser aerotransportados rápidamente en forma de bioaerosoles, a través del movimiento del aire [2].
Estos bioaerosoles son partículas sólidas suspendidas en el aire que se derivan de organismos vivos [3], incluyendo microorganismos y fragmentos de todas las variedades de materiales vivientes [4]. La supervivencia, dispersión, y reproducción de estos dependen, en gran medida, de las condiciones del entorno en que se encuentran, y de factores como la temperatura, la humedad relativa, el movimiento del aire, la luz y las fuentes de alimento [5], [6]. Los hongos, las esporas, el polen, las bacterias, los virus son algunos ejemplos de ellos [7].

En la actualidad la contaminación biológica en ambientes interiores causada por bioaerosoles, ha tomado gran importancia por diferentes sectores de nuestra sociedad, uno de ellos es el sector de la salud ya que la presencia de los microorganismos genera un riesgo biológico lo que afecta la calidad del aire intramural de las instituciones de salud, como también ofrece un riesgo potencial para la adquisición de infecciones, tanto para los pacientes, como para su familia y el personal de salud[8]. Cada vez es mayor el número de infecciones -por ejemplo, neumonía, tuberculosis, gonorrea y salmonelosis- cuyo tratamiento se vuelve más difícil debido a la pérdida de eficacia de los antibióticos. Los antibióticos son medicamentos utilizados para prevenir y tratar las infecciones bacterianas. La resistencia a los antibióticos se produce cuando las bacterias mutan en respuesta al uso de estos fármacos.

Algunas investigaciones a nivel internacional se han enfocado en el estudio de los bioaerosoles bacterianos presentes en las diversas unidades de instituciones de salud, justificado en las condiciones favorables que presentan estos ambientes para el transporte y supervivencia de microorganismos en el aire [9], 
[10]. No obstante, la resistencia bacteriana de los bioaerosoles es un tema poco estudiado y de acuerdo con la OMS se considera una de las amenazas más graves para la salud global ya que esta hace que se incrementen los costos médicos, que se prolonguen las estancias hospitalarias y que aumente la mortalidad [11].

La fuente de bioaerosoles bacterianos que causan infecciones hospitalarias puede provenir de los pacientes, del ambiente y del personal hospitalario [2]. El estudio de la concentración de bioaerosoles y la evaluación de la resistencia bacteriana a antibióticos juega un papel importante en la prevención de infecciones intrahospitalarias (IIH) y puede ser útil para el diseño de estrategias que protejan tanto a los empleados, como a los pacientes, teniendo en cuenta que cada uno de ellos tiene diferentes grados de susceptibilidad o inmunosupresión y pueden llegar a verse afectados por un mal control de estos elementos [12]. Además de proporcionar información para la investigación epidemiológica de enfermedades IIH, la investigación sobre la propagación y control de microorganismos en el aire, el monitoreo de procedimientos biológicos y su uso como medida de controlde calidad [2]

En este orden de ideas, este trabajo tiene como objetivo principal, evaluar la resistencia bacteriana de los bioaerosoles medidos en seis (6) rangos de tamaño de 0.6-7 $\mu \mathrm{m}$ en un ambiente interior de una unidad de cuidados intensivos neonatales en una institución de salud de alta complejidad en la ciudad de Barranquilla/Atlántico antes y después de las visitas familiares.

\section{Metodologia}

Se realizaron cinco monitoreos en dos ambientes diferentes durante el primer semestre del 2017 en la unidad de cuidados intensivos neonatales (UCIN) de un hospital universitario de alta complejidad, ubicado en el área metropolitana de la ciudad de Barranquilla, donde se evaluó la variación y comportamiento de las concentraciones de la carga microbiana durante dos jornadas (antes y después de la visita de los padres a los neonatos) y el alcance que tenían los bioaerosoles en la cavidad del sistema respiratorio

Las muestras de bioaerosoles bacterianos se recolectaron por triplicado esto con el objetivo de minimizar los errores y Se utilizó el Agar Plante Count dado las facultades que tiene para el crecimiento, desarrollo y contento de bacterias mesófilas, el agar se preparó según las recomendaciones del fabricante y sirvió a un $\mathrm{pH}$ de 7,0 $\pm 0,2$. Para realizar el hisopado en la recolección de las bacterias se utilizó como proteína la solución de peptona que se almaceno en tupos de ensayo.

Las muestras de aire se recolectaron usando un muestreador de aire específicamente el impactador de cascada tipo Andersen de seis etapas marca Thermo Fisher Scientific (figura 1) Este impactador se trabajó según las recomendaciones del fabricante y se recolectaron las muestras a un volumen y velocidad de 28.3 $\mathrm{L} / \mathrm{min}$ con ayuda de una bomba de vacío. Este impactador está constituido de seis placas de aluminio donde se insertan las cajas Petri para recoger las muestras; las placas del impactador presentan orificios que cuyo diámetro disminuye sucesivamente, comenzando con partículas mayores de $\geq 7,7$ $4.7,4.7-3.3,3.3-2.1,2.1-1.1$ y $1.10 \mu \mathrm{m}[13]-[17]$.

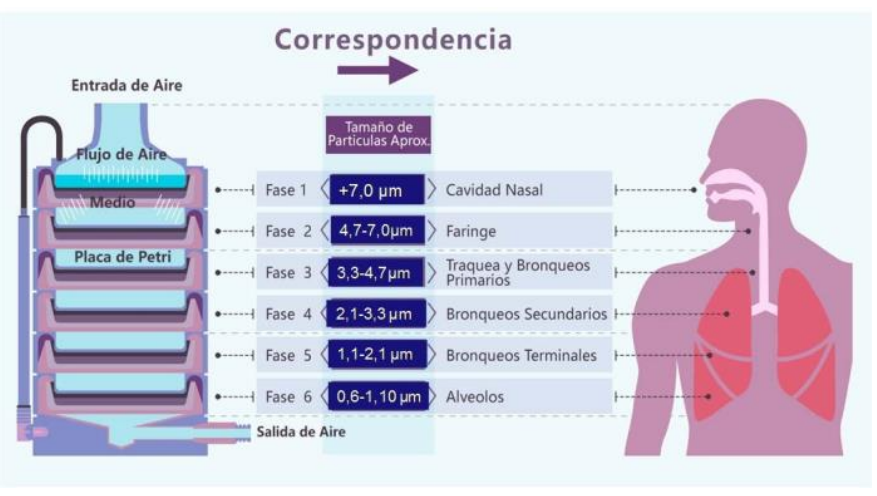

Fig. 1 Impactador por cascada de 6 etapas y su relación con el sistema respiratorio humano. Fuente: Barraza, Vargas Y (2017).

Dadas las dimensiones de la UCIN se ubicó el impactador en el eje central siendo este el lugar más representativo para la toma de muestras, la recolección de las muestras se realizó por lapso de 5 minutos tiempo optimo obtenido en el premuestreo de los tres evaluados $(3,5,10$ minutos) lo que concuerda con la literatura [13], [18]. Una vez ubicado el impactador en el punto se enciende la bomba, se calibra el volumen de aire con ayuda del Rotámetro. Cuando se establece el volumen de aire se insertan las placas Petri en cada una de las etapas del impactador y se hace impactar el aire en el medio de cultivo de las placas.

Esta metodología de tipo cuantitativa permite determinar la concentración de bioaerosoles bacterianos por metro cuadrado, dado que el número de las UFC es una de las variables que en conjunto al tiempo y caudal de aspiración da las unidades formadoras de colonia por metro cubico. La fórmula que se utilizo es la estipulada en la norma técnica de prevención de España. [19]

$$
\begin{aligned}
& \text { Concentración de bioaerosoles }\left(_{\mathrm{N}^{3}}\right) \stackrel{\mathrm{UFC}}{-} \\
& =\frac{\left(\mathrm{N}^{\circ} \mathrm{C} * 1000\right)}{\mathrm{NU}}(\mathrm{Ec.1)} \mathrm{Q} *
\end{aligned}
$$

En Donde $\mathrm{N}^{\circ} \mathrm{C}$ es la cantidad de colonias por placa, 1000 es un factor de conversión de unidades, $\mathrm{Q}$ es el caudal de aire que ingresa en el impactador de cascada $(28,3 \mathrm{~L} / \mathrm{min})$ y t el tiempo de colecta de los bioaerosoles $(5 \mathrm{~min})$

En campo también se monitorean las variables medioambientales como temperatura, sensación térmica, humedad, índice de calor y presión barométrica con la ayuda del anemómetro Kestrel 4500710830

Las muestras se incubaron por 48 horas a $37^{\circ} \mathrm{C} \pm 2$. Las colonias bacterianas se contaron y tabularon como UFC (unidad formadora de colonia). Las bacterias se identificaron por observación macroscópica (color, textura, tamaño, etc) y microscópica (morfología y tinción Gram); para la 
identificación del género y especie se utilizó BD - Phoenix ${ }^{\mathrm{TM}}$ 100 que parte de las variables microscópicas anteriormente mencionadas.

Como herramienta para la tabulación e interpretación de los datos obtenidos en campo durante los monitoreos, se utilizó una hoja de cálculo en Excel, del paquete de Microsoft Office versión 2015. En esta hoja se organizaron los datos referentes a las campañas realizadas (días de monitoreo), jornada (antes y después de las visitas), replica $(1,2$ y 3 ) y se realizaron tablas dinámicas que ayudaron en el análisis del comportamiento de los bioaerosoles. Los datos fueron analizados mediante el software Statgraphics Centurion XVI, aplicando un modelo de

regresión lineal generalizado, con el fin de determinar si existe relación entre las variables medidas (jornadas de antes y después) con las concentraciones obtenidas de los bioaerosoles bacterianos con un $95 \%$ de confianza $(\mathrm{p}<0.05)$.

Además, se realizó un análisis estadístico mediante ANOVA Multifactorial que permitió establecer si existen diferencias significativas entre los factores o variables independientes, en este caso las jornadas de antes y después

\section{RESULTADOS}

Concentraciones de Bioaerosoles bacterianos en el ambiente de la unidad de cuidados intensivos neonatal(UCIN)

\section{Concentración de bioaerosoles antes y después de visita}

El valor promedio de colonias por metro cúbico encontradas para la jornada antes (Jornada A) y después de la visita (Jornada D) fue de 14,1 y $15,5 \mathrm{UFC} / \mathrm{m}^{3}$ respectivamente, la concentración de la Jornada de después de la visita fue la que registró el mayor valor promedio. De igual manera, los resultados obtenidos se analizaron mediante un análisis de varianza (ANOVA) desarrollado a partir de las medias de las variables antes y después de la visita a los padres (jornadas) y la concentración de microorganismos (Figura 4). Teniendo en cuenta que la diferencia entre los promedios de cada grupo (jornada) no fue significativa cuando se obtuvo un valor-p mayor de 0,05 para un nivel de confianza del 95,0\% (Tabla 1).

Tabla 1. ANOVA para $U F C / \mathrm{m}^{3}$ por día de medición. Resultados obtenidos de la correlación entre los Días (antes y después) y la concentración de bioaerosoles bacterianos.

\begin{tabular}{llllll}
\hline Fuente & $\begin{array}{l}\text { Suma de } \\
\text { Cuadrados }\end{array}$ & Df & $\begin{array}{l}\text { Plaza } \\
\text { Media }\end{array}$ & $\begin{array}{l}\text { Razón- } \\
\text { F }\end{array}$ & $\begin{array}{l}\text { Valor- } \\
\text { P }\end{array}$ \\
\hline $\begin{array}{l}\text { Entre } \\
\text { Grupos }\end{array}$ & 58,82 & 1 & $\mathbf{5 8 , 8 2}$ & $\mathbf{0 , 2 2}$ & $\mathbf{0 , 6 3 9}$ \\
$\begin{array}{l}\text { Intra } \\
\text { grupos }\end{array}$ & $5,18 \mathrm{E}+04$ & 194 & 267,2 & & \\
$\begin{array}{l}\text { Total } \\
\text { (Corr.) }\end{array}$ & $5,19 \mathrm{E}+04$ & 195 & & & \\
& & & & & \\
\hline
\end{tabular}

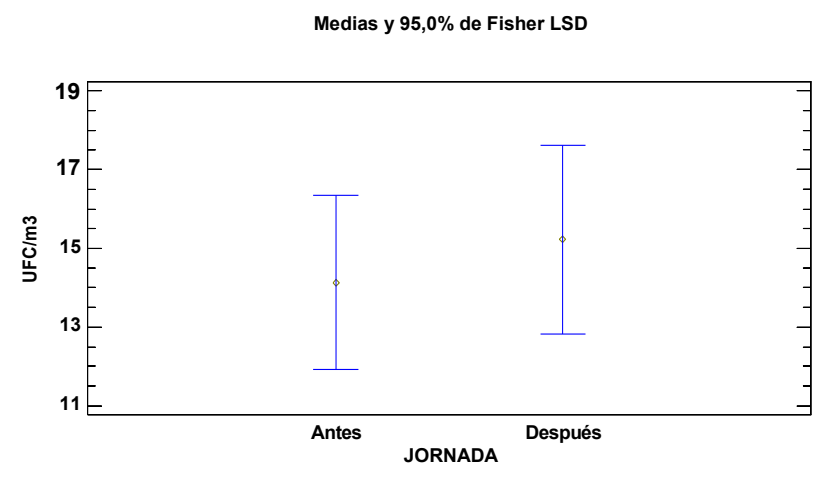

Fig. 4. Concentración por día, gráfico de medias.

\section{Concentración de bioaerosoles por campaña}

La figura 5 relaciona los resultados de la concentración en $\mathrm{UFC} / \mathrm{m}^{3}$ de bioaerosoles bacterianos durante las cinco campañas de monitoreo y las jornadas de antes y después de la visita. Las condiciones ambientales durante las cinco campañas presentaron rangos de valores de temperatura y humedad de 27 $24{ }^{\circ} \mathrm{C}$ y $74-63,2 \%$ respectivamente.

Cada campaña mostró concentraciones diferentes. En general, la máxima concentración registrada a lo largo del muestreo fue $572,44 \mathrm{UFC} / \mathrm{m}^{3}$ en la campaña tres de la Jornada $\mathrm{D}$ (jornada después de la visita), de igual modo la campaña tres de la Jornada A registró el valor más alto 508,83 UFC/m3 (jornada antes de la visitas), los resultados de la campaña tres fueron representativos para evaluar el comportamiento de los bioaerosoles ya que obtenidos los resultados de la campaña tres se realizó una vista en la institución de salud, donde se evaluó los registros de actividades clínicas y/o cambios realizados en la UCI. Los resultados reportaron que dos días antes del muestreo se realizó una jornada de limpieza en el sistema de climatización (filtros de aire acondicionado).

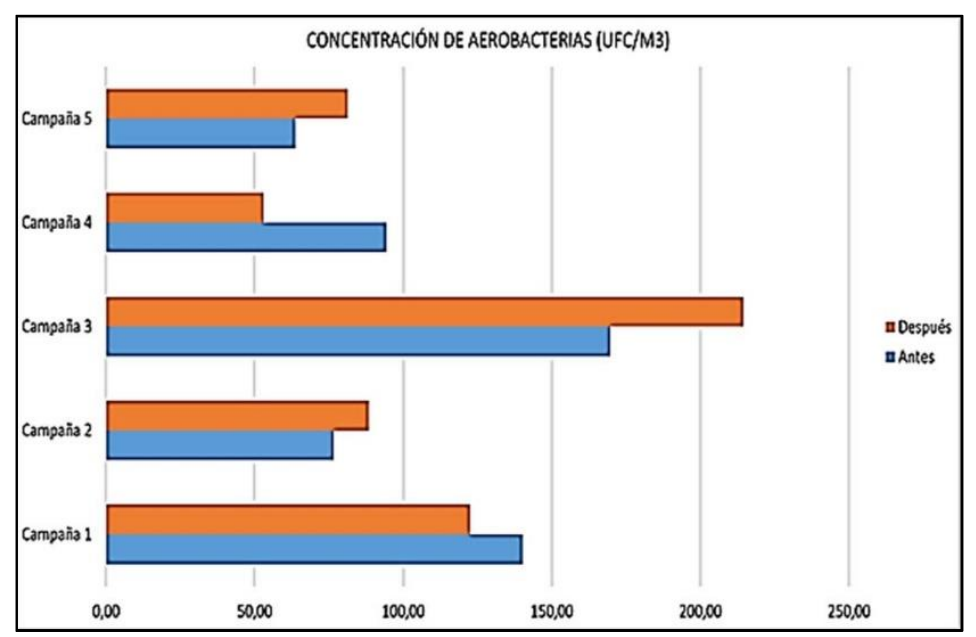

Fig. 5. Concentración total de bioaerosoles bacterianos por campaña y día. 


\section{Relación de la limpieza con la concentración bioaerosoles por campaña}

En cuanto a la campaña cuatro y cinco de la Jornada A y D se aprecia un decrecimiento en los valores de las concentraciones en comparación a la campaña uno, dos y tres, factor que puede estar asociado al proceso de limpieza de los filtros, demostrando un resultado antes y después de la limpieza. Existen investigaciones que se basan en monitorear las cargas microbiológicas del aire, antes y después de actividades de limpieza en instituciones de salud, edificios, industrias de alimentos e industrias de desechos y explican como las actividades de limpieza global, el mantenimiento de filtros de aires y el funcionamiento de otras fuentes rutinarias pueden varias la concentración de bioaerosoles en un ambiente interno [20]-[22] .En el estudio realizado por Wathes et al., (2017) se encontró que las principales fuentes en el interior para la generación de material particulado $\left(\mathrm{PM}_{10}-\mathrm{PM}_{2.5}\right)$ en relación a bioaerosoles en la UCIP (unidad de cuidados intensivos pediátricos) fueron la terapia de nebulización, la succión traqueal y las actividades de limpieza.

\section{Concentración de bioaerosoles de acuerdo a su distribución de tamaño en impactador por cascada/ sistema respiratorio humano}

La Figura 6, presenta la distribución de la concentración de bioaerosoles bacterianos obtenidos en la UCI según las etapas del impactador de cascada, se evidencia que en la $1^{\mathrm{a}}$ y $5^{\mathrm{a}}$ etapa las concentraciones de los bioaerosoles bacterianos fueron mayores, arrojando concentraciones totales de 650 y 791 $\mathrm{UFC} / \mathrm{m}^{3}$ respectivamente. De los estudios que previamente analizaron la distribución del tamaño de las bacterias en el aire mediante el uso del impactador de cascada de seis etapas, encontramos a Macher et al., [23] y Yamamoto et al., [24] que demostraron tasas de recolección más altas en la $1^{\mathrm{a}}$ y $5^{\mathrm{a}}$ etapa, ambos estudios ejecutados en espacios interiores.

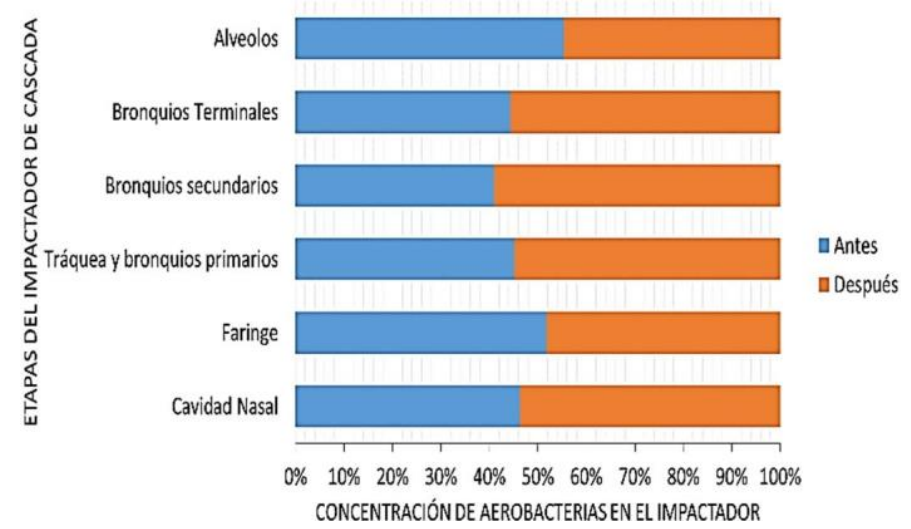

Fig. 6. Distribución de la concentración de bioaerosoles bacterianos por día y etapas del impactador.

La interpretación de la calidad del aire microbiano desde el punto de vista del tamaño de partícula es importante, ya que las partículas más pequeñas tienen una mayor probabilidad de penetrar en el sistema respiratorio [25]. Como resultado de la investigación, aproximadamente el $45,4 \%$ de las bacterias viables totales recolectadas en el impactador de casada de seis etapas estaban en el rango de tamaño de $0,65-2,1 \mu \mathrm{m}$ que pueden penetrar y depositarse en los bronquios secundarios, terminales y alveolo (etapa cuatro, cinco y seis). Por lo tanto, el 54,6\% de los bioaerosoles bacterianos se asociaron con partículas gruesas en el aire, superiores a 2,1 $\mu \mathrm{m}$, fijados principalmente en cavidad nasal, tráquea y bronquios primarios (etapa uno, dos y tres) [26], [27], [28], [29].

Los resultados reflejan una mayor concentración de bacterias cultivables en partículas gruesas; probablemente se observó este fenómeno porque existe una correlación positiva entre el material particulado en suspensión y el tamaño de las bacterias, donde las bacterias se adhieren al material en suspensión aumentando de esta manera el tamaño de la partícula que principalmente quedan alojadas en la 1a, 2a y 3a etapa [14], [27], [30]. Por otro lado, en las investigaciones de [27], [31][33] ha quedado demostrado que las fracciones de partículas finas cultivables generalmente son más altas en el ambiente interior debido a la estabilidad del aire y que el área superficial es mayor que el de las partículas gruesas, permitiendo mayor facilidad de adhesión entre las bacterias, por otro lado los bioaerosoles bacterianos en ambientes internos presentan concentraciones y patrones de distribución variables debido al tipo microorganismo, el clima local, los factores microclimáticos, el nivel de ocupación, el tipo de actividad humana, el tipo de ventilación y el mantenimiento del edificio [34]-[37]

Comparación de las concentraciones obtenidas con estándares internacionales

En general, las concentraciones de bioaerosoles bacterianos obtenidas en este trabajo podrían clasificarse como altas $(\geq 100$ $-\leq 600 \mathrm{UFC} / \mathrm{m} 3$ ), superando los índices aceptables sugeridos por la OMS, quien define que los recuentos de bacterias en el aire en salas Clase B deben ser inferiores a 100 $\mathrm{UFC} / \mathrm{m}^{3}$ [38] - [41], en esta clase se encuentran las áreas críticas que incluyen a los individuos o pacientes con inmunodepresión de la unidad de cuidados intensivos neonatal. Sin embargo, la OMS aún no ha establecido una guía específica para los niveles de concentración y los estándares actuales basados en la evaluación de riesgo para la salud todavía no son prácticos. En contraste, algunas organizaciones privadas y gubernamentales han establecido estándares cuantitativos y directrices para bioaerosoles en ambientes interiores, estos estándares se basan en valores de referencia para concentraciones de Bioaerosol, sin tener en cuenta los efectos sobre la salud humana, en este sentido, la Conferencia Americana de Higienistas Industriales Gubernamentales (ACGIH) establece que concentraciones $<100 \mathrm{UFC} / \mathrm{m}^{3}$ son consideradas Bajas, concentraciones entre 100 y $1000 \mathrm{UFC} / \mathrm{m}^{3}$ son consideradas Intermedias y concentraciones $>1000 \mathrm{UFC} / \mathrm{m}^{3}$ son consideradas Altas [23]; Edificios Internacionales Saludables establece la concentración máximo permisible $<750 \mathrm{UFC} / \mathrm{m}^{3}$ en el recuento de total de bacterias y hongos en el aire está bien si las especies no son 
infecciosas o alergénicas[43]; Asociación de Calidad del Aire Interior (IAQ) $<150 \mathrm{UFC} / \mathrm{m}^{3}$ si es una mezcla de especies no patógenas, alérgicas o tóxicas [44]; métodos de investigación en contaminación biológica del aire interior en los Países Bajos establece que una concentración $>104 \mathrm{UFC} / \mathrm{m}^{3}$ de una especie de naturaleza potencialmente patógena y concentraciones $>500$ $\mathrm{UFC} / \mathrm{m}^{3}$ en el recuento total de hongos y bacterias son consideradas amenaza para la salud, es una amenaza para la salud [45]; por su parte la Administración de Seguridad y Salud Ocupacional (OSHA) establece que concentraciones $>1000$ $\mathrm{UFC} / \mathrm{m}^{3}$ en el recuento total de hongos y bacterias Indica contaminación [46]. Los estudios detallados de bioaerosoles bacterianos en ambientes de UCI reportan intervalos de $67-$ $326 \mathrm{UFC} / \mathrm{m}^{3}, 35$ - $156 \mathrm{UFC} / \mathrm{m}^{3}, 194$ - $404 \mathrm{UFC} / \mathrm{m}^{3}, 1-423$ $\mathrm{UFC} / \mathrm{m}^{3}$ respectivamente, valores que coinciden con los encontrados en este estudio [17], [22], [32], [47].

\section{Identificación de especies}

Mediante las observaciones microscópicas y mediante el BD Phoenix $^{\mathrm{TM}} 100$ se logró determinar que los Bioaersoles bacterianos Gram positivos representaron el $92 \%$ del total de aislamientos identificados. En total, se identificaron 12 especies bacterianas diferentes, distribuidas en seis géneros: Alloiococcus, Bacillus, Kocuria, Leifsonia, Pseudomonas, Staphylococcus; encontrados en las jornadas A y D. Dentrodel género Staphylococcus se encontraron tres especies diferentes ( $S$. epidermidis, $S$. saprophyticus y $S$. cohnii ssp) mientras que para el generó Bacillus se encontraron cinco especies diferentes (B. cereus, B. megaterium, B. pumilus, B. sutiles $y$ B. thuringiensis). Los otros géneros solo presentaron una especie: Alloiococcus otitidis, Kocuria rosea, Leifsonia aquiatica y Pseudomonas pseudoalcaligene.

Además, se obtuvieron cuatro especies predominantes con concentraciones significativas. La concentración promedio más alta fue obtenida por la especie $S$. saprophyticus, esta especie había estado en todas las campañas de muestreo, seguida de $S$. epidermidis, que puede migrar desde la piel a lo largo de la superficie del dispositivo hacia el cuerpo, formando una alta Comunidad bacteriana organizada conocida como biofilm[48]. Se puede transportar al aire debido a los estafilococos en el aire que pueden ser transportados por los transportistas y permanecen viables durante mucho tiempo [49]. De manera similar, $K$. rosea fue de 8 y $28 \mathrm{UFC} / \mathrm{m}^{3}$ antes y después del tiempo de visita. El género Kocuria generalmente se considera contaminante, pero se ha documentado la participación en infecciones humanas, que pueden causar infecciones importantes, principalmente en huéspedes inmunocomprometidos $\mathrm{o}$ pacientes con enfermedades subyacentes graves como bebés neonatales[50], [51]. Además, Kocuria es una de las bacterias resistentes a la radiación[52].

\section{Concentración promedio de bioaerosoles identificados.}

se obtuvieron cuatro especies predominantes con concentraciones significativas, la mayor concentración promedio 23 y $20 \mathrm{UFC} / \mathrm{m}^{3}$ la presentó la especie $S$. saprophyticus para la jornada A y D respectivamente, estuvo presente en todas las campañas. Con un promedio de 16 $\mathrm{UFC} / \mathrm{m}^{3}$ en ambas jornadas, le sigue el $S$. epidermidis. Así mismo, se obtuvo una concentración media de la especie $K$. rosea con un promedio de 8 y $28 \mathrm{UFC} / \mathrm{m}^{3}$ se mostró en cuatro campañas de la jornada A y dos de la jornada D. Otro microorganismo con concentración media fue Bacillus cereus, presentó una concentración de 9 y $7 \mathrm{UFC} / \mathrm{m}^{3}$ también estuvo presente en cuatro campañas de la jornada A y D. En cuanto a las otras especies identificadas $A$. otitidis, $B$. megaterium, $B$. pumilus, $B$. sutiles y $B$. thuringiensis Leifsonia aquiatica y $P$. pseudoalcaligene, $S$. cohnii ssp se mantuvieron concentraciones bajas de $7 \mathrm{UFC} / \mathrm{m}^{3}$.

Staphylococcus fue el género que presentó mayor prevalencia y la respuesta a esto puede estar sujeta a que este microorganismo presenta una pared celular gruesa que proporciona una mayor tolerancia a la desecación y le permite sobrevivir por más tiempo [53].

Tabla 3 Concentración media de bacterias bacterianas por día.

\begin{tabular}{|c|c|c|c|c|c|c|c|c|c|c|c|}
\hline \multirow{2}{*}{ Microorganismo } & \multicolumn{5}{|c|}{ Antes $\left(\mathrm{UFC} / \mathbf{m}^{3}\right)$} & \multicolumn{5}{|c|}{ Después $\left(\mathrm{UFC} / \mathbf{m}^{3}\right.$ ) } & \multirow{2}{*}{$\begin{array}{l}\text { Total } \\
\text { general }\end{array}$} \\
\hline & $\begin{array}{l}\mathrm{C}- \\
1\end{array}$ & $\begin{array}{l}\mathrm{C}- \\
2\end{array}$ & $\begin{array}{l}\mathrm{C}- \\
3\end{array}$ & $\begin{array}{l}\mathrm{C}- \\
4\end{array}$ & $\begin{array}{l}\mathrm{C}- \\
5\end{array}$ & $\begin{array}{l}\mathrm{C}- \\
1\end{array}$ & $\begin{array}{l}\mathrm{C}- \\
2\end{array}$ & $\begin{array}{l}\mathrm{C}- \\
3\end{array}$ & $\begin{array}{l}\mathrm{C}- \\
4\end{array}$ & $\begin{array}{l}\mathrm{C}- \\
5\end{array}$ & \\
\hline $\begin{array}{l}\text { Alloiococcus } \\
\text { otitidis }\end{array}$ & & 7 & & & 7 & & & 7 & & & 7 \\
\hline B. cereus & 7 & 7 & 21 & & 7 & 7 & 7 & 7 & & 7 & 8 \\
\hline B. megaterium & 7 & & 7 & 7 & 7 & 7 & & 9 & & & 8 \\
\hline B. pumilus & & & 7 & & 7 & & & & 7 & 7 & 7 \\
\hline B. subtiles & 7 & & 7 & & & & 7 & & & 7 & 7 \\
\hline B. thuringiensis & 7 & & 7 & & & 7 & & 7 & & & 7 \\
\hline K. rosea & 7 & & 9 & 7 & 7 & & & 28 & 28 & & 12 \\
\hline L. aquatica & 7 & & 7 & & 7 & & & & & & 7 \\
\hline $\begin{array}{l}P . \\
\text { pseudoalcaligenes }\end{array}$ & & & 7 & & & & 7 & 7 & & & 7 \\
\hline S. cohnii ssp & 7 & & & & & & & & & & 7 \\
\hline S. epidermidis & 11 & 13 & 27 & 18 & 11 & 8 & 15 & 20 & 16 & 19 & 16 \\
\hline S. saprophyticus & 33 & 7 & 21 & & 7 & 18 & 9 & 30 & 7 & & 21 \\
\hline
\end{tabular}

\section{Fijación del microorganismo por etapa}

El S. saprophyticus y $S$. epidermidis con concentraciones relevantes lograron establecerse en las seis etapas del impactador con una predominancia en la $5^{\mathrm{a}}$ y $6^{\mathrm{a}}$ etapa, $A$. otitidis, $B$. Subtiles, $B$. thuringiensis, $K$. rosea, $P$. pseudoalcaligene, alcanzaron la última etapa del impactador recordando que esta etapa en relación con el sistema respiratorio representa la fase de los alveolos, el $S$. cohnii ssp solo se fijó en la $3^{\text {a }}$ etapa y $B$. cereus alcanzó fijarse en cinco etapas de la $1^{\mathrm{a}}$ a $5^{\mathrm{a}}$ (Figura 7). 


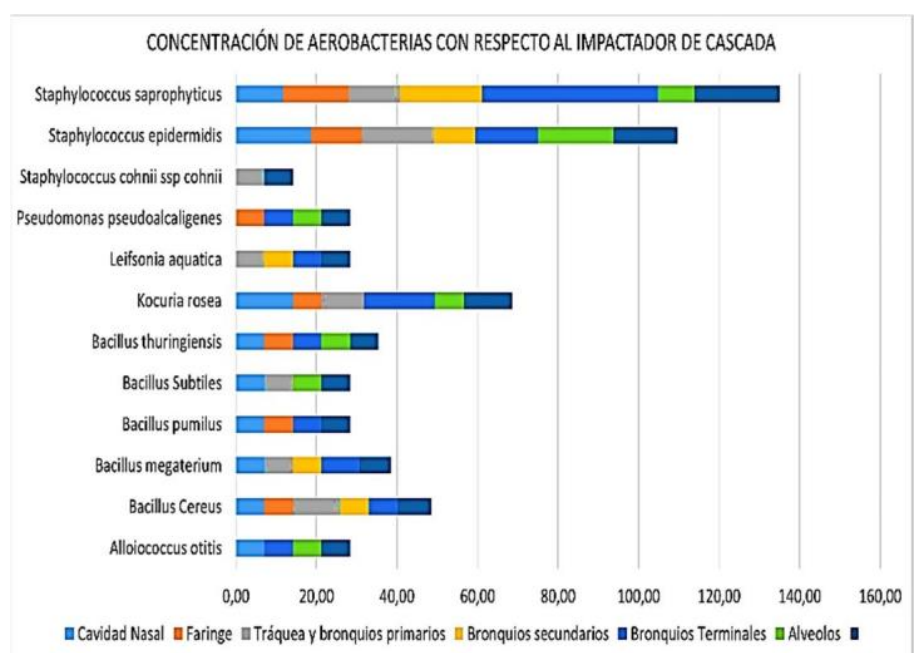

Fig. 7 Distribución de la concentración de especies bacterianas en relación con las etapas del impactador.

Algunas de las especies encontradas en la UCI fueron identificadas como patógenas agresivas y altamente peligrosas para la salud de los neonatos, capaces de causar infecciones respiratorias, neumonías e infecciones de la piel y ojos, otras especies fueron reconocidas como patógenas bajo determinadas circunstancias como es el caso del $S$. epidermidis que hace parte de flora bacteriana de la piel humana [54], [55]. Sin embargo, este microorganismo también puede emerger como patógeno intrahospitalario que infecta a pacientes inmunocomprometidos [56], [57]. Las infecciones causadas por S. epidermidis se relacionan con la colonización de cuerpos extraños (catéteres endovenosos, fístulas para hemodiálisis, marcapasos, articulaciones protésicas, injertos valvulares y/o válvulas cardiacas protésicas) y eventualmente puede producir infecciones urinarias, bacteriemia y sepsis [58].

Respecto al $S$. saprophyticus es considerado un patógeno primario a nivel urinario, agente de infección urinaria en la mujer joven y niños [59], [60]. La infección se adquiere en la comunidad, por lo tanto, el microorganismo no se considera agente de IIH [61] (Orden-Martínez et al., 2008b). No obstante, el Staphylococcus cohnii ssp si es reconocido como un patógeno u oportunista causante de IIH [62], está asociado con bacteriemia, colecistitis aguda, absceso cerebral, endocarditis, neumonía e infección del tracto urinario [63], [64].

Por otro lado, $K$. rosea, forma parte de la microbiota normal de la piel, boca y bucofarínge de los seres humanos y otros mamíferos [65]. Es un patógeno poco común y bajo ciertas circunstancias causa enfermedades en pacientes inmunocomprometidos, por lo que es considerada como oportunista [66]. En la literatura sólo existen escasos reportes médicos asociadas con catéteres vasculares, bacteriemia, colecistitis y peritonitis en pacientes crónicamente debilitados [67]. En el caso de B. cereus es un patógeno poco común pero potencialmente grave, asociado a infecciones de muy baja frecuencia, produce enfermedades de manera aislada y es reconocido principalmente como un patógeno con alta tasa de mortalidad en neonatos [68], capaz de causar meningoencefalitis hemorrágica, infecciones respiratorias, infecciones del torrente sanguíneo y afectar el sistema nervioso central de los recién nacidos prematuros [69], [70]. De igual manera, el B. pumilus ha sido identificado como un patógeno oportunista [71], [72]; rara vez causa infecciones graves y solo se ha descrito en recién nacidos e individuos inmunocomprometidos [73].

Otras especies del género Bacillus encontradas en el estudio como el B. megaterium, B. Subtilis y B. thuringiensis son comunes del suelo. El potencial patogénico de estos Bacillus generalmente se describe como bajo o ausente [74]. No obstante, se les ha reconocido cada vez más como indicadores biológicos de contaminación y pueden representar un peligro para el paciente hospitalizado o inmunosuprimido, las infecciones o enfermedades causadas por estas bacterias son raras y se reconocen pocos estudios clínicos, algunas están relacionadas con traumatismos, infecciones profundas de tejidos blandos e infecciones sistémicas [75], [76].

En el estudio también se reportó $A$. otitidis, un patógeno asociado a la otitis media con derrame (OME) enfermedad común en la infancia [77], [78], este microorganismo no es considerado patógeno oportunista o causante de enfermedades intrahospitalarias [79]. Mientras que $P$. pseudoalcaligene único Gram negativo identificado se muestra como un raro patógeno oportunista asociado a infecciones intrahospitalarias en humanos como meningitis y neumonía [80].

\section{CONCLUSIÓN}

Se obtuvieron concentraciones de bacterias viables en el aire entre 67 a $423 \mathrm{CFU} / \mathrm{m}^{3}$, con un valor promedio de $110.13 \mathrm{CFU}$ $/ \mathrm{m}^{3}$, que pueden representar una posible amenaza para la salud de los trabajadores y, por supuesto, para los bebés neonatales en la UCI. Los resultados de este estudio establecieron que no hubo diferencias estadísticamente significativas entre los dos grupos, antes y después de las visitas, para un valor de $\mathrm{p}>0.05$ y un nivel de confianza de $95.0 \%$. La concentración total para cada campaña mostró concentraciones diferentes, donde se observaron los valores más altos en la campaña 3 y los más bajos en la campaña 5 antes de la visita. Probablemente, este patrón muestra la influencia del mantenimiento de los filtros de aire, con una concentración decreciente después de que se hayan limpiado los acondicionadores de aire.

Las bacterias en el aire presentaron una distribución de tamaño homogénea para todos los rangos de tamaño. La concentración media más alta se obtuvo para los bronquios terminales y el rango de tamaño de la cavidad nasal. Aproximadamente el $46 \%$ del total de bacterias viables recolectadas estaban en el rango de tamaño de 0.6-2.1 $\mu \mathrm{m}$, lo que significa que estas partículas biológicas pueden penetrar y depositarse en los bronquios secundarios, bronquios terminales $\mathrm{y}$ alvéolos. 
En total, se identificaron 12 especies bacterianas diferentes, distribuidas en seis géneros: Alloiococcus, Bacillus, Kocuria, Leifsonia, Pseudomonas y Staphylococcus. Dentro del género Staphylococcus, se encontraron tres especies diferentes $(S$. epidermidis, $S$. saprophyticus y $S$. cohnii ssp), mientras que para los géneros Bacillus se encontraron cinco especies diferentes $(B$. cereus, B. megaterium, B. pumilus, B sutil y B. thuringiensis). Los otros géneros presentaron solo una especie: Alloiococcus otitidis, Kocuria rosea, Leifsonia aquatica y Pseudomonas pseudoalcaligene. Además, se obtuvieron cuatro especies predominantes con concentraciones significativas. La concentración promedio más alta fue obtenida por la especie $\mathrm{S}$. saprophyticus y estuvo presente en todas las campañas, seguida de S. epidermidis. De manera similar, la concentración media de la especie $K$. rosea fue de 8 y $28 \mathrm{UFC} / \mathrm{m}^{3}$ antes y después del tiempo de visita.

Staphylococcus fue el género con mayor prevalencia, probablemente debido a que este microorganismo tiene una pared celular gruesa que brinda mayor tolerancia a la desecación y le permite sobrevivir durante más tiempo. Estos hallazgos pueden ayudarnos a comprender la concentración de bacterias viables en el aire en los diferentes rangos de tamaño, así como su resistencia a los antibióticos en una UCIN. Además, nuestros resultados pueden indicar una amenaza potencial para la salud de los seres humanos debido a la resistencia a los antibióticos de las bacterias transportadas por el aire en la UCIN, considerados patógenos o patógenos en ciertas circunstancias. Sin embargo, estos datos deben interpretarse con cautela debido a las complicadas correlaciones entre las bacterias transportadas por el aire y los factores ambientales. Este es un tema importante para futuras investigaciones, por lo que se necesitan más estudios para proporcionar límites de exposición adecuados en relación con la salud en ambientes interiores de la UCIN.

\section{REFERENCIAS}

[1] E. Jara and J. Piraquive, "Determinación de la calidad de aire intrmural en la Clínica Veterinaria, Universidad de la Salle," Universidad de la Salle, Bogotá, 2016.

[2] M. Hernandez and S. León, "Determinación de la calidad del aire extramural e intramural en la sala de cirugía del hospital el tunal de la cuidad de Bogotá para el desarrollo de mecanismos de control y minimización de riesgo causado por microorganismos potencialmente nosocomiales," 2008.

[3] M. Solé and J. Obiols, "NTP 288: Síndrome del edificio enfermo: enfermedades relacionadas y papel de los bioaerosoles," Inst. Nac. Segur. e Hig. en el Trab., pp. 1-10, 2005.

[4] C. Avila Figueroa, M. Medina-mejía, and I. Hernándezramos, "Infecciones nosocomiales en una unidad de cuidados intensivos neonatales," Perinatol Reprod Hum, vol. 14, no. 14, pp. 143-150, 2000.

[5] G. Edith and C. Espinoza, "Determinación de organismos mesófilos aerobios en el ambiente de $\mathrm{Cd}$.
Obregón , Sonora; mediante el uso del monitor aéreo microbiológico y método de cuenta en placa abierta," 2005.

[6] M. J. Pelczar, E. C. . Chan, and N. R. Krieg, "Microbiology. 5th ed," Tata Mc Graw Hill Publ., 1993.

[7] J. Gutiérrez, E. Romero, L. Reyes, A. Samdoval, and C. Aguirre, "Bioaerosoles depositados vía húmeda-seca en la zona metropolitana del valle de toluca," Contancto Nucl., pp. 16-23, 2009.

[8] F. Abdel-Wahab, M. Ghoneim, M. Khashaba, A.-H. ElGilany, and D. Abdel-Hady, "Nosocomial infection surveillance in an Egyptian neonatal intensive care unit.," J. Hosp. Infect., vol. 83, no. 3, pp. 196-199, 2013.

[9] A. A. Jaffal, H. Nsanze, A. Bener, A. S. Ameen, I. M. Banat, and A. A. El Mogheth, "Hospital airborne microbial pollution in a desert country," Environ. Int., vol. 23, no. 2, pp. 167-172, 1997.

[10] K. Youn, Y. Shin, and Daekeun, "Distribution characteristics of airborne bacteria and fungi in the general hospitals of Korea.," Ind. Health, vol. 48, no. 2, pp. 236-243, 2010.

[11] W. Coronell, J. Rojas, M. Escamilla, M. Manotas, and M. Sánchez, "Infección Nosocomial En Unidades de Cuidados Intensivos Neonatales," Precop SCP, vol. 9, no. 3, pp. 30-39, 2010.

[12] M. Leung and A. H. S. Chan, "Control and management of hospital indoor air quality.," Med. Sci. Monit., vol. 12, no. 3, pp. SR17-R23, 2006.

[13] W. Wang et al., "Diversity and seasonal dynamics of airborne bacteria in the Mogao Grottoes, Dunhuang, China," Aerobiologia (Bologna)., vol. 28, no. 1, pp. 2738, 2012.

[14] C. Carvajal, "Evaluación del comportamiento de aerobacterias en el corregimiento de Cuatro," 2016.

[15] S. S. Ortiz J, Prendez M, "Utilizacion y manejo de un impactador de cascada ANDERSEN en el estudio de los aerosoles atmosfericos." Universidad de Tarapaca, Chile, Tarapaca, pp. 8-8, 1986.

[16] Y. Heo, J. Park, S. Lim, H. Hur, D. Kim, and K. Park, "Size-resolved culturable airborne bacteria sampled in rice field, sanitary landfill, and waste incineration sites," J. Environ. Monit., vol. 12, no. 8, p. 1619,2010.

[17] C. S. Li and P. A. Hou, "Bioaerosol characteristics in hospital clean rooms," Sci. Total Environ., vol. 305, no. 1-3, pp. 169-176, 2003.

[18] H. Zhu, P. Phelan, T. Duan, G. Raupp, and H. J. S. Fernando, "Characterizations and relationships between outdoor and indoor bioaerosols in an office building," China Particuology, vol. 1, no. 3, pp. 119123, 2003.

[19] M. del C. Martí Solé, "Método para el recuento de bacterias y hongos en aire," Inst. Nac. Segur. e Hig. en el Trab., vol. 3, pp. 1-4, 1991.

[20] A. Pinheiro, "MICROBIOTA FÚNGICA DO AMBIENTE DA UTI NEONATAL E DE 
AMOSTRAS CLÍNICAS DOS RECÉM-NASCIDOS INTERNADOS NO HOSPITAL UNIVERSITÁRIO DE MACEIÓ, AL," UNIVERSIDADE FEDERAL DE ALAGOAS-UFAL, 2009.

[21] C. Viegas et al., "Fungal burden in waste industry: an occupational risk to be solved," Environ. Monit. Assess., vol. 187, no. 4, 2015.

[22] C. He et al., "Particle and bioaerosol characteristics in a paediatric intensive care unit," Environ. Int., vol. 107, no. June, pp. 89-99, 2017.

[23] MACHER and J., "Sampling airborne microorganisms and aeroallergens," Air Sampl. Instruments Eval. Atmos. Contam., pp. 589-617, 1995.

[24] N. Yamamoto, D. Schmechel, B. T. Chen, W. G. Lindsley, and J. Peccia, "Comparison of quantitative airborne fungi measurements by active and passive sampling methods," J. Aerosol Sci., vol. 42, no. 8, pp. 499-507, 2011.

[25] M. Sadyś, R. Kennedy, and J. S. West, "Potential impact of climate change on fungal distributions: analysis of 2 years of contrasting weather in the UK," Aerobiologia (Bologna)., vol. 32, no. 1, pp. 127-137, 2016.

[26] R. S. Dungan, "Board-invited review: Fate and transport of bioaerosols associated with livestock operations and manures," J. Anim. Sci., vol. 88, no. 11, pp. 3693-3706, 2010.

[27] A. K. Pahari, D. Dasgupta, R. S. Patil, and S. Mukherji, "Emission of bacterial bioaerosols from a composting facility in Maharashtra, India," Waste Manag., vol. 53, pp. 22-31, 2016.

[28] W. B. Morgado Gamero et al., "Hospital Admission and Risk Assessment Associated to Exposure of Fungal Bioaerosols at a Municipal Landfill Using Statistical Models," in Intelligent Data Engineering and Automated Learning -- IDEAL 2018, 2018, pp. 210218

[29] W. B. Morgado Gamero, M. C. Ramírez, A. Parody, A. Viloria, M. H. A. López, and S. J. Kamatkar, "Concentrations and Size Distributions of Fungal Bioaerosols in a Municipal Landfill," in Data Mining and Big Data, 2018, pp. 244-253.

[30] K. Uhrbrand, A. C. Schultz, A. J. Koivisto, U. Nielsen, and A. M. Madsen, "Assessment of airborne bacteria and noroviruses in air emission from a new highlyadvanced hospital wastewater treatment plant," Water Res., vol. 112, pp. 110-119, 2017.

[31] C. Alonso, P. C. Raynor, P. R. Davies, and M. Torremorell, "Concentration, size distribution, and infectivity of airborne particles carrying swine viruses," PLoS One, vol. 10, no. 8, 2015.

[32] A. H. Awad, Y. Saeed, Y. Hassan, Y. Fawzy, and M. Osman, "Air microbial quality in certain public buildings, Egypt: A comparative study," Atmospheric Pollution Research, 2018.

[33] J. S. Pastuszka, U. Kyaw Tha Paw, D. O. Lis, A. Wlazło, and K. Ulfig, "Bacterial and fungal aerosol in indoor environment in Upper Silesia, Poland," Atmos. Environ., vol. 34, no. 22, pp. 3833-3842, 2000.

[34] R. I. Adams, M. Miletto, S. E. Lindow, J. W. Taylor, and T. D. Bruns, "Airborne bacterial communities in residences: Similarities and differences with fungi," PLoS One, vol. 9, no. 3, 2014.

[35] B. Ghosh, H. Lal, and A. Srivastava, "Review of bioaerosols in indoor environment with special reference to sampling, analysis and control mechanisms," Environ. Int., vol. 85, pp. 254-272, 2015.

[36] A. J. Prussin and L. C. Marr, "Sources of airborne microorganisms in the built environment," Microbiome, vol. 3, p. 78, 2015.

[37] K. Wai Tham, "Indoor air quality and its effects on humans-A review of challenges and developments in the last 30 years," Energy Build., vol. 130, pp. 637-650, 2016.

[38] J. Berenguer and J. L. Sanz, Cuestiones en microbiología. Editorial Hélice, 2004.

[39] S. Cabo Verde et al., "Microbiological assessment of indoor air quality at different hospital sites," Res. Microbiol., vol. 166, no. 7, pp. 557-563, 2015.

[40] M. Maldonado, J. Peña, S. De los Santos, A. Castellanos, D. Camarena, and B. Arévalo, "BIOAEROSOLES Y EVALUACIÓN DE LA CALIDAD DEL AIRE EN DOS CENTROS Laura VALDÉS-SANTIAGO , Laura J . HERNÁNDEZVALADEZ y Dora Linda GUZMÁN DE PEÑA * biopartículas INTRODUCCIÓN Los bioaerosoles o biopartículas pueden definir - se como partículas o microfragme," vol. 30, no. 4, pp. 351-363, 2014.

[41] SAMPSP, "Recomendaciones para la monitorización de la calidad microbiológica del aire (bioseguridad ambiental) en zonas hospitalarias de riesgo.," pp. 1-35, 2016.

[42] K. H. Kim, E. Kabir, and S. A. Jahan, "Airborne bioaerosols and their impact on human health," $J$. Environ. Sci. (China), vol. 67, pp. 23-35, 2018.

[43] C. Y. Rao, H. A. Burge, and J. C. Chang, "Review of quantitative standards and guidelines for fungi in indoor air.," J. Air Waste Manag. Assoc., vol. 46, no. 9, pp. 899-908, Sep. 1996.

[44] IAQA (Indoor Air Quality Association)., "Indoor Air Quality Standard \#95-1 Recommended for Florida," Longwood, Florida, 1995.

[45] H. Heida, F. Bartman, and S. C. van der Zee, "Occupational Exposure and Indoor Air Quality Monitoring in a Composting Facility," Am. Ind. Hyg. Assoc. J., vol. 56, no. 1, pp. 39-43, Jan. 1995.

[46] OSHA - Occupational Safety and Health Administration., Indoor air quality. Canada, 1994, pp. 15968-16039.

[47] K. Y. Kim and C. N. Kim, "Airborne microbiological characteristics in public buildings of Korea," Build. Environ., vol. 42, no. 5, pp. 2188-2196, 2007.

[48] Y. Zhang et al., "Synergy of ambroxol with 
vancomycin in elimination of catheter-related Staphylococcus epidermidis biofilm in vitro and in vivo," J. Infect. Chemother., vol. 21, no. 11, pp. 808$815,2015$.

[49] K. Hardjawinata, R. Setiawati, and W. Dewi, "Bactericidal efficacy of ultraviolet irradiation on Staphylococcus aureus," Asian J. Oral Maxillofac. Surg., vol. 17, no. 3, pp. 157-161, 2005.

[50] K. M. Sohn, J. Y. Baek, S. H. Kim, S. Cheon, and Y. S. Kim, "Catheter-related bacteremia caused by kocuria salsicia: The first case," J. Infect. Chemother., vol. 21, no. 4, pp. 305-307, 2015.

[51] A. Uekotter, J. Konig, G. Peters, and K. Becker, Portinfection due to Kocuria rhizophila in an 8 year old child with methylmalonic aciduria. 2006.

[52] M. Gholami, Z. Etemadifar, and M. Bouzari, "Isolation a new strain of Kocuria rosea capable of tolerating extreme conditions," J. Environ. Radioact., vol. 144, pp. 113-119, 2015.

[53] M. De La Rosa, M. . Mosso, and C. Ullán, "El aire: hábitat y medio de transmisión de microorganismos," Obs. Medioambient., vol. 5, pp. 375-402, 2002.

[54] B. Martinez, R. Ruiz, and R. Perez, "[What are we learning about Staphylococcus saprophyticus?]," Enferm. Infecc. Microbiol. Clin., vol. 26, no. 8, pp. 495-499, 2008.

[55] W. Ziebuhr, S. Hennig, M. Eckart, H. Kränzler, C. Batzilla, and S. Kozitskaya, "Nosocomial infections by Staphylococcus epidermidis: how a commensal bacterium turns into a pathogen," Int. J. Antimicrob. Agents, vol. 28, no. SUPPL. 1, pp. 14-20, 2006.

[56] Y. Dong and C. P. Speer, "The role of Staphylococcus epidermidis in neonatal sepsis: Guarding angel or pathogenic devil?," International Journal of Medical Microbiology, vol. 304, no. 5-6. pp. 513-520, 2014.

[57] K. A. Thompson, A. M. Bennett, and J. T. Walker, "Aerosol survival of Staphylococcus epidermidis," J. Hosp. Infect., vol. 78, no. 3, pp. 216-220, 2011.

[58] S. Borrego, I. Perdomo, J. De La Paz, S. Gómez De Saravia, and P. Guiamet, "Relevamiento microbiológico del aire y de materiales almacenados en el Archivo Histórico del Museo de La Plata, Argentina y en el Archivo Nacional de la República de Cuba," Rev. del Mus. La Plata, vol. 18, no. 119, pp. 1-18, 2011.

[59] M. Macedo and J. Blanco, "Infecciones hospitalarias," Control, pp. 245-254, 2008.

[60] B. Orden-Martínez, R. Martínez-Ruiz, and R. MillánPérez, "¿Qué estamos aprendiendo de Staphylococcus saprophyticus?," Enferm. Infecc. Microbiol. Clin., vol. 26, no. 8, pp. 495-499, 2008.

[61] S. Estrada, M. Restrepo, E. Jaramillo, and C. M. Montoya, "Staphylococcus saprophyticus como agente etiológico de la infección del tracto urinario," Acta médica Colomb., vol. 15, no. 5, pp. 292-297, 1990.

[62] J. Soldera, W. L. Nedel, P. R. Cardoso, and P. A. d'Azevedo, "Bacteremia due to Staphylococcus cohnii ssp. urealyticus caused by infected pressure ulcer: case report and review of the literature," Sao Paulo Med.J., vol. 131, no. 1806-9460 (Electronic), pp. 59-61, 2013.

[63] S. Mendoza et al., "Draft genome sequences of two opportunistic pathogenic strains of Staphylococcus cohnii isolated from human patients," Stand. Genomic Sci., vol. 12, no. 1, 2017.

[64] Z. Shahandeh, H. Shafi, and F. Sadighian, "Association of staphylococcus cohnii subspecies urealyticum infection with recurrence of renal staghorn stone," $J$. Intern. Med., vol. 6, no. 1, pp. 40-42, 2015.

[65] M. Paul, R. Gupta, S. Khush, and R. Thakur, "Kocuria rosea: An emerging pathogen in acute bacterial meningitis- Case report," J. Microbiol. Antimicrob. Agents, vol. 1, no. 1, pp. 4-7, 2015.

[66] J. S. Moreira, A. G. L. Riccetto, M. T. N. da Silva, and M. M. dos S. Vilela, "Endocarditis by Kocuria rosea in an immunocompetent child," Brazilian J. Infect. Dis., vol. 19, no. 1, pp. 82-84, 2015.

[67] M. Corti, M. F. Villafañe, I. Soto, O. Palmieri, and R. Callejo, "Bacteriemia por Kocuria rosea en un paciente con SIDA," Rev. Chil. infectología, vol. 29, no. 3, pp. 355-356, 2012.

[68] N. J. Hilliard, R. L. Schelonka, and K. B. Waites, "Bacillus cereus bacteremia in a preterm neonate," $J$. Clin. Microbiol., vol. 41, no. 7, pp. 3441-3444, 2003.

[69] C. Machado, A. Silva, M. J. Magalhães, C. Sá, and E. Abreu, "Severe Bacillus cereus infection in a neonatal intensive care unit," Case Rep. Perinat. Med, vol. 3, no. 2, pp. 159-162, 2014.

[70] S. Puvabanditsin, A. Zaafran, E. Garrow, R. Diwan, D. Mehta, and N. Phattraprayoon, "Bacillus cereus meningoencephalitis in a neonate," Hong Kong J. Paediatr., vol. 12, no. 4, pp. 293-296, 2007.

[71] V. M. Shivamurthy, S. Gantt, C. Reilly, P. Tilley, J. Guzman, and L. Tucker, "Bacillus pumilus Septic Arthritis in a Healthy Child," Can. J. Infect. Dis. Med. Microbiol., 2016.

[72] Y. Yuan and M. Gao, "Genomic analysis of a ginger pathogen Bacillus pumilus providing the understanding to the pathogenesis and the novel control strategy," Sci. Rep., vol. 5, 2015.

[73] M. Kimouli et al., "Two cases of severe sepsis caused by Bacillus pumilus in neonatal infants," J. Med. Microbiol., vol. 61, no. 4, pp. 596-599, 2012.

[74] F. Celandroni et al., "Identification and pathogenic potential of clinical Bacillus and Paenibacillus isolates," PLoS One, vol. 11, no. 3, 2016.

[75] R. Camacho et al., "Characterization of Cry toxins from autochthonous Bacillus thuringiensis isolates from Mexico," Bol. Med. Hosp. Infant. Mex., vol. 74, no. 3, pp. 193-199, 2017.

[76] M. R. Oggioni, G. Pozzi, P. E. Valensin, P. Galieni, and C. Bigazzi, "Recurrent septicemia in an immunocompromised patient due to probiotic strains of Bacillus subtilis.," J. Clin. Microbiol., vol. 36, no. 1, pp. 325-6, 1998. 
[77] A. F. Sheikh et al., "Identification of Alloiococcus otitidis, Streptococcus pneumoniae, Moraxella catarrhalis and Haemophilus influenzae in children with otitis media with effusion," Jundishapur J. Microbiol., vol. 8, no. 3, 2015.

[78] K. Tano, R. Von Essen, P. O. Eriksson, and A. Sjöstedt, "Alloiococcus otitidis - Otitis media pathogen or normal bacterial flora?," APMIS, vol. 116, no. 9, pp. 785-790, 2008

[79] A. Harimaya, R. Takada, Y. Somekawa, N. Fujii, and T. Himi, "High frequency of Alloiococcus otitidis in the nasopharynx and in the middle ear cavity of otitisprone children," Int. J. Pediatr. Otorhinolaryngol., vol. 70, no. 6, pp. 1009-1014, 2006.

[80] J. E. Hage, P. E. Schoch, and B. A. Cunha, "Pseudomonas pseudoalcaligenes peritoneal dialysisassociated peritonitis," Perit. Dial. Int., vol. 33, no. 2, pp. 223-224, 2013.

[81] J. Potorski, I. Koniuszewska, M. Czatzkowska, and M. Harnisz, "Drug resistance in airborne bacteria isolated from waste management and wastewater treatment plants in Olsztyn," vol. 66, pp. 1-8, 2019.

[82] S. United, S. Environmental, and S. California, "Continuous and Semicontinuous Monitoring Techniques for Particulate Matter Mass and Chemical Components: A Synthesis of Findings from EPA' $s$ Particulate Matter Supersites Program an ... Continuous and Semicontinuous Monitoring Techniques for Particulate," no. May 2016, 2008.
[83] R. N. Jones, "Microbial Etiologies of Hospital< Acquired Bacterial Pneumonia and Ventilator< Associated Bacterial Pneumonia," Clin. Infect. Dis., vol. 51, no. S1, pp. S81-S87, 2010.

[84] U. Valle and U. Valle, "La resistencia de bacterias a antibióticos , antisépticos y desinfectantes una manifestación de los mecanismos de supervivencia y adaptación," 2007.

[85] Secretaría Distrital de Salud de Bogotá, "Boletín epidemiológico de resistencia bacteriana - SIVIBAC año 2007," Grup. para el Control la Resist. Bact. Bogotá, 2009.

[86] A. Patricia, L. Isabel, S. Milena, and M. Victoria, "Vigilancia de infecciones asociadas a la atención en salud, resistencia bacteriana y consumo de antibióticos en hospitales de alta complejidad , Colombia , 2011," 2014.

[87] A. Hoyos Orrego, O. Rivera Rivera, C. Hoyos Posada, C. Mesa Restrepo, and V. Alfaro., "Características clínicas, epidemiológicas y de susceptibilidad a los antibióticos en casos de bacteriemia por Klebsiella pneumoniae en neonatos," CES, vol. 21, pp. 31-39, 2007.

[88] GREBO, "S ECRETARIA MANUAL DE ACTUALIZACION EN RESISTENCIA BACTERIANA Y NORMAS CLSI M100 - S20," Secr. salud, pp. 1-78, 2010. 